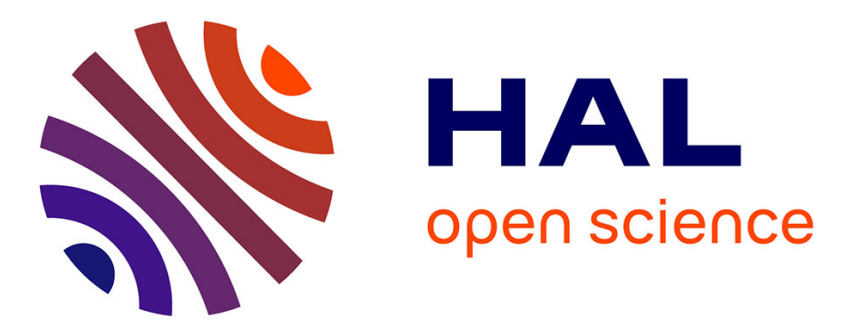

\title{
Non-Covalent Interactions using Local Correlation Methods: Energy Partitioning, Geometry Optimisation and Harmonic Frequency Calculations
}

Jamie Platts, J. Grant Hill

\section{- To cite this version: \\ Jamie Platts, J. Grant Hill. Non-Covalent Interactions using Local Correlation Methods: Energy Partitioning, Geometry Optimisation and Harmonic Frequency Calculations. Molecular Physics, 2010, 108 (11), pp.1497-1504. 10.1080/00268971003757977 . hal-00602607}

\section{HAL Id: hal-00602607 \\ https://hal.science/hal-00602607}

Submitted on 23 Jun 2011

HAL is a multi-disciplinary open access archive for the deposit and dissemination of scientific research documents, whether they are published or not. The documents may come from teaching and research institutions in France or abroad, or from public or private research centers.
L'archive ouverte pluridisciplinaire $\mathbf{H A L}$, est destinée au dépôt et à la diffusion de documents scientifiques de niveau recherche, publiés ou non, émanant des établissements d'enseignement et de recherche français ou étrangers, des laboratoires publics ou privés. 


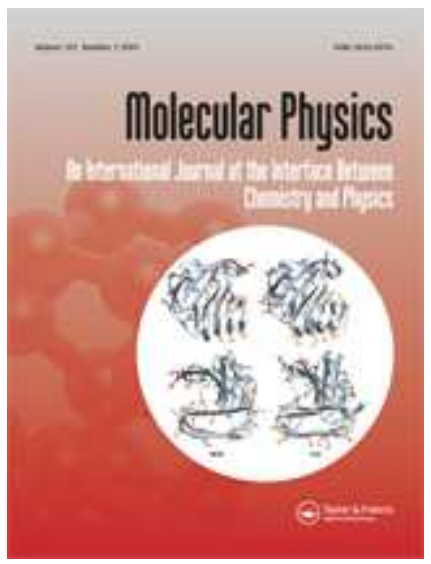

\section{Non-Covalent Interactions using Local Correlation Methods: Energy Partitioning, Geometry Optimisation and Harmonic Frequency Calculations}

\begin{tabular}{|r|l|}
\hline Journal: & Molecular Physics \\
\hline Manuscript ID: & TMPH-2010-0047.R1 \\
\hline Manuscript Type: & Full Paper \\
\hline Date Submitted by the \\
Author: & 04-Mar-2010 \\
\hline Complete List of Authors: & $\begin{array}{l}\text { Platts, Jamie; Cardiff University, Chemistry } \\
\text { Hill, J.; Washington State University, Chemistry }\end{array}$ \\
\hline Keywords: & $\begin{array}{l}\text { Local correlation, Non-covalent interactions, Hydrogen bonding, Pi- } \\
\text { stacking, Zero point enrgy }\end{array}$ \\
\hline
\end{tabular}

\section{今 ScholarONE \\ Manuscript Central}




\begin{abstract}
The performance of density fitting, local correlation methods (DF-LMP2 and DF-LCCSD) in studies of non-covalent interactions is tested against literature data for a standard set of 22 intermolecular complexes. Partitioning of interaction energy in the local correlation approach, based on the classes of occupied and virtual orbital involved in the interaction, clearly distinguishes the three types of interaction present in the set of complexes, in agreement with previous classifications. Geometry optimisation is found to be straightforward with DF-LMP2 without the need for counterpoise correction, resulting in geometries very close to previous, counterpoisecorrected structures. Spin-component scaling of gradients to correct for the known shortcomings of conventional MP2 has only a small effect on geometries in most cases, but significantly alters the distance between aromatic rings in stacked complexes. Harmonic frequency calculation is made possible by efficient use of parallel computing resources, and confirms all structures to be true minima, unlike previous estimates using density functional theory. Corrections for the change in zero-point vibrational energy are determined from this data, and typically constitute between 10 and $50 \%$ of the overall binding energy of the complex.
\end{abstract}




\section{Introduction}

Non-covalent interactions play an important part in a wide range of chemical and biological phenomena, for instance folding of proteins and nucleic acids into their correct 3-dimensional shapes. ${ }^{1}$ Intermolecular forces acting within molecular clusters are widely used as models of such non-covalent interactions, since their effects can be more easily separated from intra-molecular forces. Ab initio theoretical methods are increasingly used to complement experimental study of such clusters. The majority of theoretical treatments of non-covalent interactions employ the supermolecular approach, in which interaction energy is calculated as the difference in energy between the cluster and its constituent monomers. However, two significant drawbacks of this approach are the need for large orbital basis sets, including polarisation and diffuse functions, for proper description of all interactions between molecules, and the related problem of basis set superposition error (BSSE). ${ }^{2}$

These problems can be ameliorated, to a large extent, by use of local correlation methods, which effectively eliminate BSSE by restricting excitations from occupied orbitals to those virtual orbitals that are in close spatial proximity. ${ }^{3}$ Moreover, in combination with the density fitting (DF) approximation, methods such as DF-LMP2 ${ }^{4}$ and DF-LCCSD ${ }^{5,6,7,8}$ make significant efficiency savings over conventional calculations, approaching linear scaling in favourable cases, hence allowing application of suitable orbital basis sets for description of non-covalent interactions. A further advantage of the local correlation approach is that intermolecular correlation energy can be partitioned into physically meaningful contributions, such as dispersion and ionic terms, on the basis of the occupied and virtual orbitals involved. ${ }^{3}$ This approach is complementary to decomposition schemes, and is based solely on the local correlation treatment of the cluster and so requires no further calculations or significant computational resources.

BSSE can also be accounted for by use of the counterpoise method, ${ }^{9}$ but for a cluster of $n$ molecules, this procedure requires $(n+1)$ energy calculations in the full basis set of the cluster, substantially increasing the computational resources required. The situation becomes still less satisfactory if geometry optimisation of the cluster is a goal, as separate gradient calculations for the cluster and all fragments are necessary. Without such corrections, geometry optimisation is likely to result in underestimation of intermolecular separations and overestimation of stabilities. Alternative approaches, such as symmetry adapted perturbation theory (SAPT) eliminate BSSE by construction, but are currently limited to pairs of molecules. ${ }^{10}$ 


\section{Computational Methods}

All calculations were performed with the MOLPRO suite of programs, ${ }^{16}$ employing the correlation consistent aug-cc-pVTZ orbital basis set throughout. ${ }^{17,18}$ Density fitting of MP2 energies employed the corresponding MP2FIT basis set, ${ }^{19}$ while the underlying Hartree-Fock calculation also employed density fitting ${ }^{20}$ with the corresponding JKFIT basis. ${ }^{21}$ DF in LCCSD for integrals over three and four external orbitals used the larger aug-cc-pVQZ/MP2FIT auxiliary basis sets to minimize fitting errors. The localised orbitals required for local correlation methods were generated via the Pipek-Mezey method, ${ }^{22}$ while the orbital domain selection followed the 
procedure of Boughton and Pulay, ${ }^{23}$ with merging of rotationally invariant $\pi$-domains where appropriate. Initial test calculations indicated that using domains calculated at large intermolecular separation and then frozen for calculations on the interacting system produced almost negligible differences in geometry and harmonic frequencies when compared with domains computed solely for the interacting system (so-called floating domains). For example, with the small methane dimer system a maximum difference of $6 \mathrm{~cm}^{-1}$ between normal modes was observed, while for the larger parallel-displaced benzene dimer system the maximum difference was $0.2 \mathrm{~cm}^{-1}$. As deviations of this size are likely to be small compared with both the basis set and method errors, floating domains were used for all geometry optimisations and calculation of numerical harmonic frequencies.

Analytical gradients for DF-LMP2 methods $^{24}$ are available in MOLPRO, enabling efficient geometry optimisation. All geometry optimisations reported commenced from the reported literature structure, and employed default criteria for convergence. Calculation of root mean square (RMS) deviation between initial and final structures used Chemcraft. ${ }^{25}$ Following geometry optimisation, harmonic frequencies were calculated by numerical differentiation of analytic gradients using central differences, employing the default step size of 0.01 a.u. This is a sizeable task for the larger molecules considered, but was made feasible by use of a parallel algorithm, ${ }^{26}$ as implemented in MOLPRO, in which individual energy calculations on different geometry displacements used in the formation of gradients and hessians are performed independently on separate processors. Individual DF-LMP2 calculations did not take account of molecular symmetry (as this usually leads to symmetry equivalent localised orbitals), but this numerical algorithm can use symmetry to reduce the number of displacements required. The local character of the orbitals allows for the partitioning of the intermolecular interaction energy into intramolecular, dispersive, and ionic components of the correlation energy. ${ }^{3}$ This is achieved by dividing the system into monomers and separating the excitations from localised orbitals into different classes based on the domains the pair of electrons are excited into.

\section{Results and Discussion}

Table 1 presents the results of partitioning the DF-LMP2 correlation energy into its intermolecular components, along with the interaction energy at the SCF level, for the S22 set of complexes of Hobza and co-workers. ${ }^{15}$ Following reference 3, dispersion refers to simultaneous excitations on different monomers, ionic to excitation from an occupied orbital on one monomer to a virtual 
orbital on the other along with a single excitation on the acceptor monomer, and dispersionexchange to cross-excitations. Such definitions stem naturally from the local correlation approach, but may not be directly comparable to assignments from methods such as SAPT. ${ }^{10}$ Intramolecular correlation can also be calculated in this fashion, but does not show significant differences from the sum of monomer correlation energies. The three intermolecular correlation terms are taken directly from the DF-LMP2 cluster calculation, while the SCF interaction energy was calculated using the supermolecular approach. In all cases, dispersion-exchange energies are essentially negligible, and are included only for completeness.

Table 1 SCF interaction energies and partitioned DF-LMP2 intermolecular energies $\left(\mathrm{kcal} \mathrm{mol}^{-1}\right)$

\begin{tabular}{|c|c|c|c|c|}
\hline & SCF & Dispersion & Ionic & $\begin{array}{l}\text { Dispersion- } \\
\text { Exchange }\end{array}$ \\
\hline Ammonia dimer & -1.27 & -1.23 & -0.77 & -0.01 \\
\hline Water dimer & -3.72 & -1.06 & -1.23 & +0.01 \\
\hline Formic acid dimer & -15.49 & -4.20 & -5.63 & +0.01 \\
\hline Formamide dimer & -12.32 & -3.73 & -4.09 & +0.08 \\
\hline Uracil dimer HB & -9.31 & -4.82 & -4.79 & +0.07 \\
\hline 2-Pyridoxine...2-aminopyridine & -5.81 & -5.52 & -4.76 & -0.03 \\
\hline Adenine... Thymine HB & -6.40 & -5.68 & -4.79 & +0.01 \\
\hline Methane dimer & +0.35 & -0.73 & -0.14 & 0.00 \\
\hline Ethene dimer & +0.79 & -1.88 & -0.60 & +0.01 \\
\hline Benzene...methane & +1.60 & -2.19 & -0.86 & +0.02 \\
\hline Benzene dimer stack & +6.33 & -7.22 & -2.78 & +0.02 \\
\hline Pyrazine dimer & +7.60 & -7.77 & -3.11 & +0.03 \\
\hline Uracil dimer stack & +5.20 & -9.15 & -3.13 & +0.01 \\
\hline Indole... benzene stack & +8.62 & -10.60 & -4.46 & +0.11 \\
\hline Adenine... Thymine stack & +5.72 & -14.05 & -5.42 & +0.05 \\
\hline Ethene...ethyne & -0.52 & -1.01 & -0.58 & 0.01 \\
\hline Benzene...water & -0.35 & -2.01 & -1.05 & +0.01 \\
\hline Benzene... ammonia & +0.74 & -2.10 & -0.95 & +0.01 \\
\hline Benzene...cyanide & -1.55 & -2.75 & -1.63 & +0.04 \\
\hline Benzene dimer $\mathrm{T}$ & +2.54 & -3.84 & -1.57 & +0.05 \\
\hline Indole...benzene $\mathrm{T}$ & +2.00 & -5.13 & -2.55 & +0.05 \\
\hline
\end{tabular}


The first seven clusters, denoted as hydrogen bonded in the original work, all display significant stabilisation at the SCF level, reflecting the importance of electrostatics in such interactions. Nonetheless, dispersion and ionic correlation effects contribute significantly to the overall stabilisation, with approximately equal importance. In all seven cases, the SCF stabilisation is the largest single term, but in three (ammonia dimer, 2-pyridoxine...2-aminopyridine and adenine...thymine) the combined correlation terms outweigh the SCF contribution. For example, in Watson-Crick hydrogen bonded adenine...thymine the SCF, dispersion and ionic contributions are $39 \%, 35 \%$ and $29 \%$ of the overall binding energy, respectively.

The next eight complexes were denoted as predominantly bound through dispersion, ${ }^{15}$ an assignment supported by the present analysis. In all eight clusters, SCF energies are repulsive, with all stabilisation stemming from correlation effects. Unlike the hydrogen bonded complexes, dispersion effects are much greater (typically by a factor of two to five) than ionic ones in these complexes, more than counteracting the repulsive SCF term. This is exemplified by the archetypal stacked, parallel-displaced benzene dimer, for which dispersion effects are 2.5 times greater than ionic ones. The final seven complexes are of so-called "mixed" type, with no single contribution dominating. This designation is again supported by the current analysis; SCF energies are attractive in three cases and repulsive in the remaining four. Dispersion contributions are generally larger than ionic ones, but not to the same extent as for the dispersion-bound clusters. Complexes that could be designated as containing $\mathrm{X}-\mathrm{H} \ldots \pi(\mathrm{X}=\mathrm{C}, \mathrm{N}, \mathrm{O})$ non-covalent bonds, such as benzene...water, benzene...ammonia and benzene...HCN, show significant differences from the conventional hydrogen bonds. Most notably, dispersion contributions are significantly greater than SCF ones. The clear contrast in the origin of stabilisation between the three classes of complex is illustrated in Figure 1. 


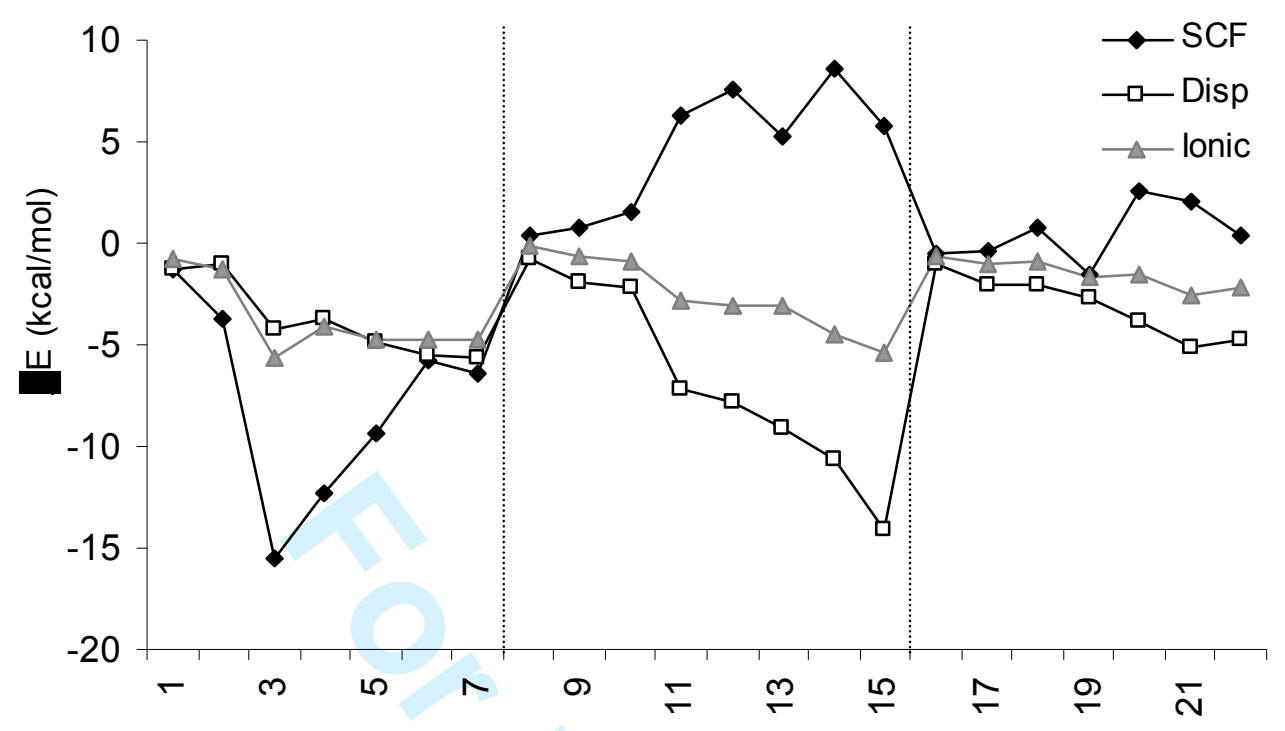

Figure 1 Illustration of energy partitioning data from Table 1. Vertical dashed lines show separation of classes of complex used in ref. 15. The numbering on the abscissa corresponds to the ordering of the complexes in Table 1.

The analysis of Table 1 is based on MP2 methods, known to perform adequately for H-bonding but to substantially over-estimate interaction energies of stacked complexes. Analogous partitioning of correlation energy with the DF-LCCSD method (energy partitioning of perturbative triples contributions is not currently possible) has also been performed. For inclusion of all relevant electron pairs in the LCCSD treatment, modification of the default distance based domain selection criteria is required: after some trial and error, all orbital pairs within a distance of 8 a.u. are treated as strong pairs, i.e., at the CCSD level. Unfortunately, this renders DF-LCCSD calculations of the larger complexes unfeasible with a moderately large basis set such as aug-cc-pVTZ.

The partitioning of the DF-LCCSD correlation energy is presented in Table 2 for selected, smaller complexes, and shows that the trends observed using DF-LMP2 methods are largely preserved at this improved level of theory. For dispersion bound and mixed complexes, the tendency of MP2 to overestimate interaction energies is evident in both dispersion and ionic terms, with rather larger reductions in the former than the latter. This is exemplified in data for the ethene dimer, for which dispersion contributions are reduced by $0.1 \mathrm{kcal} \mathrm{mol}^{-1}$ and ionic by $0.02 \mathrm{kcal} \mathrm{mol}^{-1}$. This compares well with the $\Delta \operatorname{CCSD}(\mathrm{T})$ correction to the overall interaction of +0.09 reported in ref 15 . 
Table 2 Partitioned DF-LCCSD interaction energies for selected complexes $\left(\mathrm{kcal} \mathrm{mol}^{-1}\right)$

\begin{tabular}{lccc}
\hline Name & Dispersion & Ionic & $\begin{array}{c}\text { Dispersion- } \\
\text { Exchange }\end{array}$ \\
\hline Ammonia dimer & -1.29 & -0.79 & -0.01 \\
Water dimer & -1.20 & -1.24 & -0.03 \\
Methane dimer & -0.72 & -0.16 & 0.00 \\
Ethene dimer & -1.78 & -0.58 & 0.00 \\
Benzene...methane & -2.03 & -0.82 & +0.01 \\
Ethene...ethyne & -0.95 & -0.54 & 0.00 \\
Benzene....water & -1.90 & -0.99 & -0.01 \\
Benzene....ammonia & -1.96 & -0.85 & -0.01 \\
\hline
\end{tabular}

This analysis was performed at the literature geometries, which were obtained with either MP2 or $\operatorname{CCSD}(\mathrm{T})$ optimisation with triple- or quadruple- $\zeta$ basis sets and including counterpoise correction. This can be a laborious process, and might be significantly eased by the advantages of the DFLMP2 method discussed above, particularly the removal of BSSE. Table 3 reports the results of full, unconstrained geometry optimisation of the same S22 complexes using DF-LMP2/aug-ccpVTZ, starting from the literature geometry. Proper application of local correlation methods requires symmetry to be disabled, but the optimisation algorithm retains any elements of symmetry present in the initial geometry.

Optimisation generally proceeded smoothly, in most cases reaching the default convergence criteria in MOLPRO (maximum gradient below $3 \times 10^{-4}$ a.u.) in less than 10 steps. Table 3 demonstrates that the resulting optimised geometries are very similar to their literature equivalents in all but one case, that being the phenol dimer. Performance for hydrogen bonded and dispersion bound complexes is similar, whereas the slightly larger RMS deviation for the mixed complexes is almost entirely due to the phenol dimer (omitting this point gives a mean RMS of $0.018 \AA$ for the remaining six complexes). No significant difference in the quality of agreement is observed between MP2 and $\operatorname{CCSD}(\mathrm{T})$ optimised literature geometries. Figure 2 shows the literature and optimised geometries of the phenol dimer, which by inspection are highly similar. The hydrogen bond O...H distances in the two cases are almost identical (1.937 vs. $1.935 \AA$ ). However, the dihedral angle between the mean planes of each molecule are different, with values of $60.5^{\circ}$ in the literature geometry and $56.3^{\circ}$ in the DF-LMP2 optimised structure. Thus, DF-LMP2 brings the 
Table 3 RMS deviations from literature geometry $(\AA)$

\begin{tabular}{|c|c|c|}
\hline Name & DF-LMP2 & SCSN \\
\hline Ammonia dimer $^{a}$ & 0.025 & 0.027 \\
\hline Water dimer ${ }^{a}$ & 0.030 & 0.037 \\
\hline Formic acid dimer $^{\mathrm{a}}$ & 0.017 & 0.016 \\
\hline Formamide dimer $^{\text {a }}$ & 0.025 & 0.028 \\
\hline Uracil dimer HB & 0.008 & 0.026 \\
\hline 2-Pyridoxine...2-aminopyridine & 0.009 & 0.034 \\
\hline Adenine...Thymine HB & 0.011 & 0.028 \\
\hline Methane dimer ${ }^{a}$ & 0.005 & 0.050 \\
\hline Ethene dimer ${ }^{a}$ & 0.017 & 0.049 \\
\hline Benzene...methane & 0.005 & 0.039 \\
\hline Benzene dimer stack & 0.029 & 0.068 \\
\hline Pyrazine dimer & 0.021 & 0.048 \\
\hline Uracil dimer stack & 0.023 & 0.053 \\
\hline Indole... benzene stack & 0.017 & 0.039 \\
\hline Adenine... Thymine stack & 0.018 & 0.035 \\
\hline Ethene...ethyne ${ }^{\text {a }}$ & 0.014 & 0.017 \\
\hline Benzene...water & 0.026 & 0.012 \\
\hline Benzene... ammonia & 0.019 & 0.021 \\
\hline Benzene...cyanide & 0.019 & 0.016 \\
\hline Benzene dimer $\mathrm{T}$ & 0.015 & 0.027 \\
\hline Indole...benzene $\mathrm{T}$ & 0.012 & 0.023 \\
\hline Phenol dimer & 0.183 & 0.173 \\
\hline Mean & 0.025 & 0.039 \\
\hline Mean H-bond & 0.018 & 0.028 \\
\hline Mean dispersion & 0.017 & 0.048 \\
\hline
\end{tabular}




\begin{tabular}{lll}
\hline Mean Mixed & 0.041 & 0.041 \\
Mean $\operatorname{CCSD}(\mathrm{T}){ }^{\mathrm{b}}$ & 0.019 & 0.032
\end{tabular}

${ }^{\mathrm{a}}$ Literature geometry from CP-corrected CCSD(T)/AVQZ optimisation; all others from CPcorrected MP2/AVTZ. ${ }^{\mathrm{b}}$ For literature geometries optmised with $\operatorname{CCSD}(\mathrm{T})$ only.

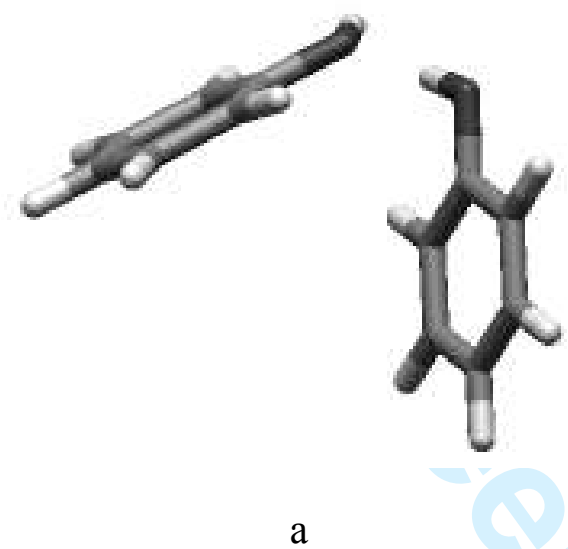

a

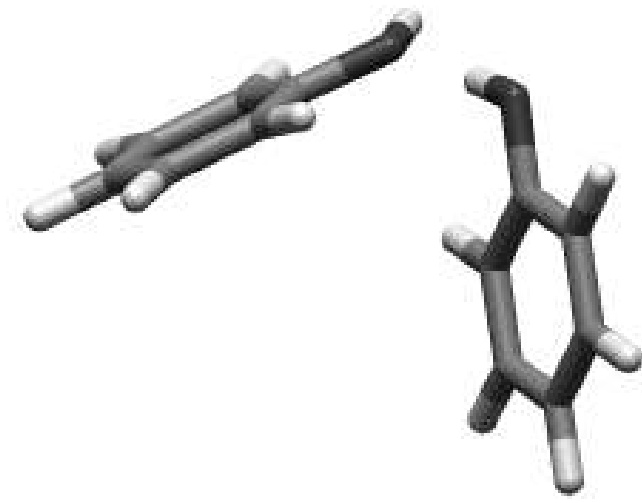

b

Figure 2 Phenol dimer a) literature geometry; b) DF-LMP2 optmised.

Both conventional MP2 and DF-LMP2 are well known to overestimate the interaction energy of stacked complexes, and might therefore be expected to underestimate intermolecular separation in such complexes. This is apparent in potential energy curves for the stacked benzene dimer, which reach a minimum at vertical separation of $c a .3 .5 \AA$ at $\mathrm{CBS}(\mathrm{T})$ level and $c a .3 .35 \AA$ at $\mathrm{MP} 2{ }^{27}$ Recently, it was demonstrated that a new parameterisation of SCS designed specifically for noncovalent interactions and denoted SCSN, ${ }^{13 a}$ overcomes this deficiency, resulting in high-accuracy interaction energies for all classes of complex with errors of less than $0.5 \mathrm{kcal} \mathrm{mol}^{-1}$. In this approach, correlation energy from same- and opposite-spin electron pairs are weighted by scaling factors in order to correct for systematic errors in standard MP2. The same scaling factors can be applied to calculation of analytic gradients, allowing optimisation to minima on this scaled potential energy surface. Table 3 also reports the RMS deviations from literature geometry using this approach.

In general, SCSN optimised structures are slightly further from literature values than unscaled DFLMP2, both for MP2 and CCSD(T) reference data. Overall performance is similar to unscaled optimisation for hydrogen bonded and mixed complexes, but differences are much larger for dispersion bound complexes. The relatively small RMS deviations reported in Table 3 mask some 
rather more significant changes; for instance, in the stacked benzene dimer, the separation of mean planes is $3.361 \AA$ in the literature (MP2/TZ-CP optimised) geometry, whereas our SCSN optimisation results in a separation of $3.487 \AA$, much closer to the minimum of the CBS(T) curve. ${ }^{27}$ A similar trend is apparent in other stacked complexes, albeit to a lesser extent: in the pyrazine dimer, the molecular planes are separated by $3.261 \AA$ in literature geometry and $3.270 \AA$ in the SCSN geometry. In stacked adenine-thymine, molecular planes are not quite parallel, so for the current investigation the perpendicular distance is defined as that from the mean plane of adenine to the centroid of the 6-membered ring of thymine, which is $3.174 \AA$ in literature and $3.205 \AA$ in SCSN optimised geometry. Thus, it is evident that the excess stabilisation of stacked complexes at MP2 level carries through into potential energy surfaces, and can be simply and efficiently corrected by the empirical SCSN scheme.

A second SCSN geometry optimisation was performed for the T-shaped benzene dimer, following the recent study by Wang et al. that showed the lowest energy geometry of this complex to deviate from the ideal $C_{2 \mathrm{v}}$ geometry. ${ }^{28}$ Starting from a slightly offset geometry, optimisation proceeded smoothly to a point just $0.06 \mathrm{kcal} \mathrm{mol}^{-1}$ lower in energy than that reported in Table 3 . Shown in Figure 3, the SCSN geometry is similar to that reported in ref.28, with the donor $\mathrm{C}-\mathrm{H}$ directed towards a $\mathrm{C}-\mathrm{C}$ bond rather than the centre of the acceptor ring.
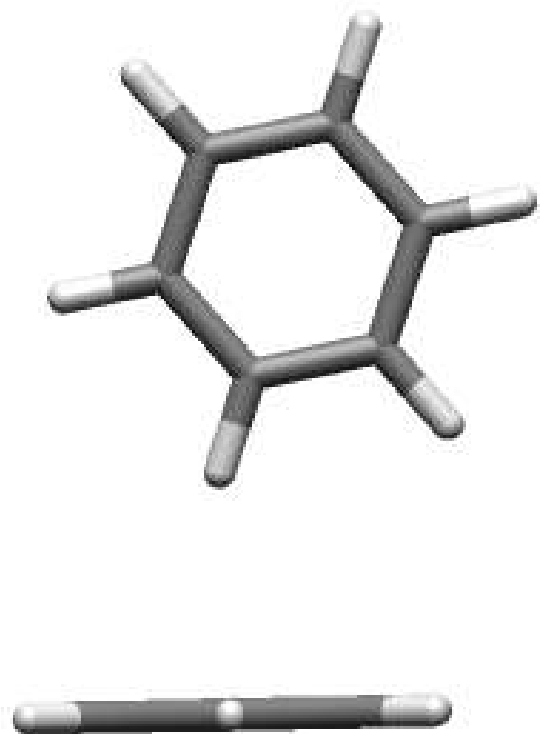

Figure 3 SCSN optimised geometry of T-shaped benzene dimer

The fully optimised DF-LMP2 geometries were then used to calculate harmonic frequencies using numerical differentiation. Firstly, and most importantly, this procedure confirms that all but one 
optimised structures are true minima at this theoretical level, as confirmed by the lack of imaginary frequencies. As noted above, any molecular symmetry present in the literature geometry was retained in these calculations, such that our data lends support to the symmetries employed in the original study of Hobza and co-workers. This is in marked contrast to a recent DFT-based study of the same set of molecules, in which one or more imaginary frequencies were observed for several complexes. $^{29}$ The only exception is the T-shaped benzene dimer, for which the high-symmetry $\left(C_{2 \mathrm{v}}\right)$ form is found to be a transition state, with a single imaginary frequency of $-10.8 \mathrm{~cm}^{-1}$. Reoptimisation with lower $\left(C_{\mathrm{s}}\right)$ symmetry results in a geometry similar to that shown in Figure 3, which is found to be a true minimum on the potential energy surface.

A similar procedure was followed for the SCSN-optimised geometries. In this case, both the $\mathrm{C}_{2 \mathrm{v}}$ and $\mathrm{C}_{\mathrm{s}}$ optimised forms of the T-shaped benzene dimer are found to be true minima, echoing the findings of Wang et $a l,{ }^{28}$ who used dispersion-corrected DFT to show that both forms are stable and are connected by a transition state with a barrier of just $0.01 \mathrm{kcal} \mathrm{mol}^{-1}$. In this regard, SCSN again seems to be slightly more accurate than unscaled DF-LMP2 in finding both forms to be stable, but the inherent errors associated with this approach are much larger than these energy differences, such that any conclusion based on this data must be tentative at best. All remaining structures are found to be minima using this approach. Two geometries of the stacked (paralleldisplaced) benzene dimer were tested, one with a $\mathrm{C}-\mathrm{H}$ bond situated over the partner benzene and a second with a $\mathrm{C}-\mathrm{C}$ bond over the other benzene. Both are found to be minima using SCSN, with effectively identical energies within the expected accuracy of the method (less than $0.1 \mathrm{kcal}$ $\mathrm{mol}^{-1}$ difference).

As well as confirming structures as minima, harmonic frequency calculations can be used to estimate the change in zero-point vibrational energy (ZPVE) that accompanies formation of a complex from constituent monomers. Such changes stem largely from conversion of translational and rotational degrees of freedom of individual molecules into intermolecular vibrations. Such vibrations are likely to be strongly anharmonic in nature, such that harmonic data can only be a first-order approximation to the true ZPVE correction. Nonetheless, obtaining even harmonic vibrational data for the larger complexes considered here is a considerable challenge, and the data presented in Table 4 is a step towards establishing accurate ZPVE corrections for systems of this size. 
Table 4 Harmonic zero point energy corrections for binding $\left(\mathrm{kcal} \mathrm{mol}^{-1}\right)$

\begin{tabular}{|c|c|c|c|}
\hline Name & DF-LMP2 & Scaled $^{\mathrm{a}}$ & SCSN \\
\hline Ammonia dimer & 1.70 & 1.62 & 1.66 \\
\hline Water dimer & 2.37 & 2.27 & 2.33 \\
\hline Formic acid dimer & 2.08 & 1.99 & 2.06 \\
\hline Formamide dimer & 2.64 & 2.53 & 2.52 \\
\hline Uracil dimer HB & 1.21 & 1.16 & 1.15 \\
\hline 2-Pyridoxine...2-aminopyridine & 1.01 & 0.97 & 0.98 \\
\hline Adenine...thymine HB & 0.95 & 0.90 & 0.89 \\
\hline Methane dimer & 1.16 & 1.11 & 1.14 \\
\hline Ethene dimer & 0.83 & 0.80 & 0.82 \\
\hline Benzene...methane & 0.64 & 0.62 & 0.70 \\
\hline Benzene dimer stack & 0.53 & 0.51 & 0.56 \\
\hline Pyrazine dimer & 0.61 & 0.58 & 0.51 \\
\hline Uracil dimer stack & 0.58 & 0.56 & 0.56 \\
\hline Indole...benzene stack & 0.25 & 0.24 & 0.43 \\
\hline Adenine...thymine stack & 0.72 & 0.68 & 0.61 \\
\hline Ethene...ethyne & 0.71 & 0.68 & 0.73 \\
\hline Benzene...water & 1.06 & 1.01 & 1.04 \\
\hline Benzene... ammonia & 0.70 & 0.67 & 0.79 \\
\hline Benzene...cyanide & 0.60 & 0.57 & 0.71 \\
\hline Benzene dimer $\mathrm{T}$ & 0.52 & 0.49 & 0.67 \\
\hline Indole... benzene $\mathrm{T}$ & 0.62 & 0.60 & 0.59 \\
\hline Phenol dimer & 0.95 & 0.91 & 0.99 \\
\hline
\end{tabular}

${ }^{\text {a }}$ Scaled from raw DF-LMP2 data using the recommended scaling factor of 0.956

$\triangle$ ZPVE values on formation of complexes from monomers are largest for the hydrogen bonded complexes, and are in general rather smaller for dispersion bound and mixed complexes. The single largest change is found for the formamide dimer, closely followed by water and formic acid dimers. The value for the water dimer is similar to previous estimates using MP2 methods with the harmonic approximation $\left(2.1-2.3 \mathrm{kcal} \mathrm{mol}^{-1}\right)$, and as expected rather larger than values that take anharmonicity into account $\left(1.8-2.1 \mathrm{kcal} \mathrm{mol}^{-1}\right){ }^{30}$ The larger aromatic systems containing two strong hydrogen bonds show much smaller $\triangle \mathrm{ZPVE}$, falling to $c a .1 \mathrm{kcal} \mathrm{mol}^{-1}$ for 2-pyridoxine...2- 
aminopyridine and adenine...thymine. Values for dispersion bound and mixed complexes are in general rather smaller than for hydrogen bonded ones. For example, $\triangle \mathrm{ZPVE}$ for the stacked uracil dimer is less than half the value for the hydrogen bonded isomer, with a similar pattern for the adenine-thymine complexes. A slightly surprising exception to this pattern is the methane dimer, whose $\triangle \mathrm{ZPVE}$ value of $1.16 \mathrm{kcal} \mathrm{mol}^{-1}$ is more than twice the binding energy. This may represent the breakdown of the harmonic approximation, or be a result of noise introduced by the use of numerical differences in such a weakly bound case.

In addition to raw DF-LMP2 data, Table 4 also contains $\triangle$ ZPVE values scaled by the value of 0.956 recommended by Hrenar et $a l,{ }^{11}$ and values from numerical frequency calculation based on SCSN energies. Changes using the single scaling factor are generally very small, and do not significantly alter the conclusions drawn from unscaled data. A similar pattern emerges from most of the SCSN data, with one exception: in the stacked indole-benzene complex, the SCSN value is almost double that from unscaled DF-LMP2. In the absence of benchmark data, it is not possible to decide which of these values is the more reliable, but in the context of other stacked complexes the raw DF-LMP2 value seems rather small.

\section{Conclusions}

The utility of local correlation methods, when combined with density fitting, has been demonstrated in the study of non-covalent interactions. By construction, these methods allow for decomposition of correlation energy into contributions from dispersion and ionic excitations, thus giving insight into the origin of stabilisation at no additional computational cost to the correlation calculation. This analysis, at both DF-LMP2 and DF-LCCSD levels, clearly distinguishes complexes bound by hydrogen bonding, dispersion, or a mixture of these effects. The lack of basis set superposition error in local methods, coupled with their computational efficiency, makes them ideally suited for simple geometry optimisation and harmonic frequency calculation. Geometry optimisation leads to structures in close agreement with published, counterpoise-corrected geometries, demonstrating the utility of this approach. Harmonic frequency analysis, using both unscaled and SCSN-scaled MP2 data, indicates that most complexes within the S22 set are at least local minima on their respective potential energy surfaces. The only exception to this conclusion is the $\mathrm{C}_{2 \mathrm{v}}$ T-shaped benzene dimer, which is a minimum with SCSN scaling but a saddle point with unscaled MP2. Zero-point vibrational energy corrections to binding energies have also been 
estimated, using raw data and two distinct scaling methods. In general, all three methods are in reasonable agreement, with corrections of between $20 \%$ and $50 \%$ of uncorrected binding energies.

\section{Acknowledgements}

This work was funded by the UK EPSRC, grant ref. EP/C013328/1.

\section{References}

${ }^{1}$ A. Pavlov, P.M. Mitrasinovic, Curr. Org. Chem., 2010, 14, 129.

2 P. Hobza, H.L. Selzle, E.W. Schlag, J. Phys. Chem., 1996, 100, 18790.

${ }^{3}$ M. Schütz, G. Rauhut, H.-J. Werner, J. Phys. Chem. A, 1998, 102, 5997.

${ }^{4}$ H.-J. Werner, F. R. Manby, P. J. Knowles, J. Chem. Phys., 2003, 118, 8149.

${ }^{5}$ C. Hampel, H.-J. Werner, J. Chem. Phys., 1996, 104, 6286.

${ }^{6}$ M. Schütz, H.-J. Werner, J. Chem. Phys., 2001, 114, 661.

${ }^{7}$ M. Schütz, Phys. Chem. Chem. Phys., 2002, 4, 3941.

${ }^{8}$ M. Schütz, F. R. Manby, Phys. Chem. Chem. Phys., 2003, 5, 3349.

${ }^{9}$ S. F. Boys, F. Bernardi, Mol. Phys., 1970, 19, 553.

${ }^{10}$ B. Jeziorski, R. Moszynski, K. Szalewicz, Chem. Rev., 1994, 94, 1887.

${ }^{11}$ T. Hrenar, G. Rauhut, H.-J. Werner, J. Phys. Chem. A, 2006, 110, 2060.

${ }^{12}$ S. Grimme, J. Chem. Phys., 2003, 118, 9095.

13 a) J.G. Hill, J.A. Platts, J. Chem. Theor. Comput., 2007, 3, 80. b) R.A. Bachorz, F. A. Bischoff, S. Hofener, W. Klopper, P. Ottiger, R. Leist, J. A. Frey, S. Leutwyler, Phys. Chem. Chem. Phys., 2008, 10, 2758.

${ }^{14}$ O. Marchetti and H.-J. Werner, J. Phys. Chem. A, 2009, 113, 11580.

${ }^{15}$ P. Jurečka, J. Šponer, J. Černý, P. Hobza, Phys. Chem. Chem. Phys., 2006, 8, 1985.

${ }^{16}$ MOLPRO, version 2008.1, a package of ab initio programs, H.-J. Werner, P. J. Knowles, R. Lindh, F. R. Manby, M. Schütz and others, see http://www.molpro.net.

${ }^{17}$ T. Dunning Jr., J. Chem. Phys., 1989, 90, 1007.

${ }^{18}$ R. A. Kendall, T. Dunning Jr., R. J. Harrison, J. Chem. Phys., 1991, 96, 6797.

${ }^{19}$ F. Weigend, A. Köhn, C. Hättig, J. Chem. Phys., 2002, 116, 3175.

${ }^{20}$ R. Polly, H.-J. Werner, F. R. Manby, P. J. Knowles, Mol. Phys., 2004, 102, 2311.

${ }^{21}$ F. Weigend, Phys. Chem. Chem. Phys., 2002, 4, 4285.

${ }^{22}$ J. Pipek, P. G. Mezey, J. Chem. Phys., 1989, 90, 4916.

${ }^{23}$ J. W. Boughton, P. Pulay, J. Comput. Chem., 1993, 14, 736. 
${ }^{24}$ M. Schütz, H.-J. Werner, R. Lindh, F. R. Manby, J. Chem. Phys., 2004, 121, 737.

${ }^{25}$ Chemcraft v1.6: www.chemcraftprog.com

${ }^{26}$ J. Neugebauer, M. Reiher, C. Kind, B.A. Hess, J. Comput. Chem., 2002, 23, 895.

27 a) S. Tsuzuki, K. Honda, T. Uchimaru, M. Mikami, K. Tanabe, J. Am. Chem. Soc., 2000, 122, 3746. b) M. O. Sinnokrot, C. D. Sherrill, J. Am. Chem. Soc., 2004, 126, 7690. c) J.G. Hill, J.A. Platts, H.-J. Werner, Phys. Chem. Chem. Phys., 2006, 8, 4072.

${ }^{28}$ W. Wang, M. Pitoňák, P. Hobza, ChemPhysChem, 2007, 8, 2107.

${ }^{29}$ K. Gkionis, J.G. Hill, S.P. Oldfield, J.A. Platts, J. Mol. Model., 2009, 15, 1051.

${ }^{30}$ M. W. Feyereisen, D. Feller, D.A. Dixon, J. Phys. Chem., 1996, 100, 2993. 


\title{
Non-Covalent Interactions using Local Correlation Methods: Energy Partitioning, Geometry Optimisation and Harmonic Frequency Calculations
}

James A. Platts* and J. Grant Hill ${ }^{\dagger}$

School of Chemistry, Cardiff University, Park Place, Cardiff CF10 3AT, UK

${ }^{\dagger}$ Present address: Department of Chemistry, Washington State University, Pullman, Washington 99164-4630, USA.

* Author for correspondence:

Phone: +44-2920-874950

FAX: +44-2920-874030

E-mail: platts@cardiff.ac.uk

\begin{abstract}
The performance of density fitting, local correlation methods (DF-LMP2 and DF-LCCSD) in studies of non-covalent interactions is tested against literature data for a standard set of 22 intermolecular complexes. Partitioning of interaction energy in the local correlation approach, based on the classes of occupied and virtual orbital involved in the interaction, clearly distinguishes the three types of interaction present in the set of complexes, in agreement with previous classifications. Geometry optimisation is found to be straightforward with DF-LMP2 without the need for counterpoise correction, resulting in geometries very close to previous, counterpoisecorrected structures. Spin-component scaling of gradients to correct for the known shortcomings of conventional MP2 has only a small effect on geometries in most cases, but significantly alters the distance between aromatic rings in stacked complexes. Harmonic frequency calculation is made possible by efficient use of parallel computing resources, and confirms all structures to be true minima, unlike previous estimates using density functional theory. Corrections for the change in zero-point vibrational energy are determined from this data, and typically constitute between 10 and $50 \%$ of the overall binding energy of the complex.
\end{abstract}




\section{Introduction}

Non-covalent interactions play an important part in a wide range of chemical and biological phenomena, for instance folding of proteins and nucleic acids into their correct 3-dimensional shapes. ${ }^{1}$ Intermolecular forces acting within molecular clusters are widely used as models of such non-covalent interactions, since their effects can be more easily separated from intra-molecular forces. Ab initio theoretical methods are increasingly used to complement experimental study of such clusters. The majority of theoretical treatments of non-covalent interactions employ the supermolecular approach, in which interaction energy is calculated as the difference in energy between the cluster and its constituent monomers. However, two significant drawbacks of this approach are the need for large orbital basis sets, including polarisation and diffuse functions, for proper description of all interactions between molecules, and the related problem of basis set superposition error (BSSE). ${ }^{2}$

These problems can be ameliorated, to a large extent, by use of local correlation methods, which effectively eliminate BSSE by restricting excitations from occupied orbitals to those virtual orbitals that are in close spatial proximity. ${ }^{3}$ Moreover, in combination with the density fitting (DF) approximation, methods such as DF-LMP2 ${ }^{4}$ and DF-LCCSD ${ }^{5,6,7,8}$ make significant efficiency savings over conventional calculations, approaching linear scaling in favourable cases, hence allowing application of suitable orbital basis sets for description of non-covalent interactions. A further advantage of the local correlation approach is that intermolecular correlation energy can be partitioned into physically meaningful contributions, such as dispersion and ionic terms, on the basis of the occupied and virtual orbitals involved. ${ }^{3}$ This approach is complementary to decomposition schemes, and is based solely on the local correlation treatment of the cluster and so requires no further calculations or significant computational resources.

BSSE can also be accounted for by use of the counterpoise method, ${ }^{9}$ but for a cluster of $n$ molecules, this procedure requires $(n+1)$ energy calculations in the full basis set of the cluster, substantially increasing the computational resources required. The situation becomes still less satisfactory if geometry optimisation of the cluster is a goal, as separate gradient calculations for the cluster and all fragments are necessary. Without such corrections, geometry optimisation is likely to result in underestimation of intermolecular separations and overestimation of stabilities. Alternative approaches, such as symmetry adapted perturbation theory (SAPT) eliminate BSSE by construction, but are currently limited to pairs of molecules. ${ }^{10}$ 


\section{Computational Methods}

All calculations were performed with the MOLPRO suite of programs, ${ }^{16}$ employing the correlation consistent aug-cc-pVTZ orbital basis set throughout. ${ }^{17,18}$ Density fitting of MP2 energies employed the corresponding MP2FIT basis set, ${ }^{19}$ while the underlying Hartree-Fock calculation also employed density fitting ${ }^{20}$ with the corresponding JKFIT basis. ${ }^{21}$ DF in LCCSD for integrals over three and four external orbitals used the larger aug-cc-pVQZ/MP2FIT auxiliary basis sets to minimize fitting errors. The localised orbitals required for local correlation methods were generated via the Pipek-Mezey method, ${ }^{22}$ while the orbital domain selection followed the 
procedure of Boughton and Pulay, ${ }^{23}$ with merging of rotationally invariant $\pi$-domains where appropriate. Initial test calculations indicated that using domains calculated at large intermolecular separation and then frozen for calculations on the interacting system produced almost negligible differences in geometry and harmonic frequencies when compared with domains computed solely for the interacting system (so-called floating domains). For example, with the small methane dimer system a maximum difference of $6 \mathrm{~cm}^{-1}$ between normal modes was observed, while for the larger parallel-displaced benzene dimer system the maximum difference was $0.2 \mathrm{~cm}^{-1}$. As deviations of this size are likely to be small compared with both the basis set and method errors, floating domains were used for all geometry optimisations and calculation of numerical harmonic frequencies.

Analytical gradients for DF-LMP2 methods $^{24}$ are available in MOLPRO, enabling efficient geometry optimisation. All geometry optimisations reported commenced from the reported literature structure, and employed default criteria for convergence. Calculation of root mean square (RMS) deviation between initial and final structures used Chemcraft. ${ }^{25}$ Following geometry optimisation, harmonic frequencies were calculated by numerical differentiation of analytic gradients using central differences, employing the default step size of 0.01 a.u. This is a sizeable task for the larger molecules considered, but was made feasible by use of a parallel algorithm, ${ }^{26}$ as implemented in MOLPRO, in which individual energy calculations on different geometry displacements used in the formation of gradients and hessians are performed independently on separate processors. Individual DF-LMP2 calculations did not take account of molecular symmetry (as this usually leads to symmetry equivalent localised orbitals), but this numerical algorithm can use symmetry to reduce the number of displacements required. The local character of the orbitals allows for the partitioning of the intermolecular interaction energy into intramolecular, dispersive, and ionic components of the correlation energy. ${ }^{3}$ This is achieved by dividing the system into monomers and separating the excitations from localised orbitals into different classes based on the domains the pair of electrons are excited into.

\section{Results and Discussion}

Table 1 presents the results of partitioning the DF-LMP2 correlation energy into its intermolecular components, along with the interaction energy at the SCF level, for the S22 set of complexes of Hobza and co-workers. ${ }^{15}$ Following reference 3, dispersion refers to simultaneous excitations on different monomers, ionic to excitation from an occupied orbital on one monomer to a virtual 
orbital on the other along with a single excitation on the acceptor monomer, and dispersionexchange to cross-excitations. Such definitions stem naturally from the local correlation approach, but may not be directly comparable to assignments from methods such as SAPT. ${ }^{10}$ Intramolecular correlation can also be calculated in this fashion, but does not show significant differences from the sum of monomer correlation energies. The three intermolecular correlation terms are taken directly from the DF-LMP2 cluster calculation, while the SCF interaction energy was calculated using the supermolecular approach. In all cases, dispersion-exchange energies are essentially negligible, and are included only for completeness.

Table 1 SCF interaction energies and partitioned DF-LMP2 intermolecular energies $\left(\mathrm{kcal} \mathrm{mol}^{-1}\right)$

\begin{tabular}{|c|c|c|c|c|}
\hline & SCF & Dispersion & Ionic & $\begin{array}{l}\text { Dispersion- } \\
\text { Exchange }\end{array}$ \\
\hline Ammonia dimer & -1.27 & -1.23 & -0.77 & -0.01 \\
\hline Water dimer & -3.72 & -1.06 & -1.23 & +0.01 \\
\hline Formic acid dimer & -15.49 & -4.20 & -5.63 & +0.01 \\
\hline Formamide dimer & -12.32 & -3.73 & -4.09 & +0.08 \\
\hline Uracil dimer HB & -9.31 & -4.82 & -4.79 & +0.07 \\
\hline 2-Pyridoxine...2-aminopyridine & -5.81 & -5.52 & -4.76 & -0.03 \\
\hline Adenine...Thymine HB & -6.40 & -5.68 & -4.79 & +0.01 \\
\hline Methane dimer & +0.35 & -0.73 & -0.14 & 0.00 \\
\hline Ethene dimer & +0.79 & -1.88 & -0.60 & +0.01 \\
\hline Benzene...methane & +1.60 & -2.19 & -0.86 & +0.02 \\
\hline Benzene dimer stack & +6.33 & -7.22 & -2.78 & +0.02 \\
\hline Pyrazine dimer & +7.60 & -7.77 & -3.11 & +0.03 \\
\hline Uracil dimer stack & +5.20 & -9.15 & -3.13 & +0.01 \\
\hline Indole... benzene stack & +8.62 & -10.60 & -4.46 & +0.11 \\
\hline Adenine...Thymine stack & +5.72 & -14.05 & -5.42 & +0.05 \\
\hline Ethene...ethyne & -0.52 & -1.01 & -0.58 & 0.01 \\
\hline Benzene...water & -0.35 & -2.01 & -1.05 & +0.01 \\
\hline Benzene...ammonia & +0.74 & -2.10 & -0.95 & +0.01 \\
\hline Benzene...cyanide & -1.55 & -2.75 & -1.63 & +0.04 \\
\hline Benzene dimer $\mathrm{T}$ & +2.54 & -3.84 & -1.57 & +0.05 \\
\hline Indole...benzene $\mathrm{T}$ & +2.00 & -5.13 & -2.55 & +0.05 \\
\hline
\end{tabular}


The first seven clusters, denoted as hydrogen bonded in the original work, all display significant stabilisation at the SCF level, reflecting the importance of electrostatics in such interactions. Nonetheless, dispersion and ionic correlation effects contribute significantly to the overall stabilisation, with approximately equal importance. In all seven cases, the SCF stabilisation is the largest single term, but in three (ammonia dimer, 2-pyridoxine...2-aminopyridine and adenine...thymine) the combined correlation terms outweigh the SCF contribution. For example, in Watson-Crick hydrogen bonded adenine...thymine the SCF, dispersion and ionic contributions are $39 \%, 35 \%$ and $29 \%$ of the overall binding energy, respectively.

The next eight complexes were denoted as predominantly bound through dispersion, ${ }^{15}$ an assignment supported by the present analysis. In all eight clusters, SCF energies are repulsive, with all stabilisation stemming from correlation effects. Unlike the hydrogen bonded complexes, dispersion effects are much greater (typically by a factor of two to five) than ionic ones in these complexes, more than counteracting the repulsive SCF term. This is exemplified by the archetypal stacked, parallel-displaced benzene dimer, for which dispersion effects are 2.5 times greater than ionic ones. The final seven complexes are of so-called "mixed" type, with no single contribution dominating. This designation is again supported by the current analysis; SCF energies are attractive in three cases and repulsive in the remaining four. Dispersion contributions are generally larger than ionic ones, but not to the same extent as for the dispersion-bound clusters. Complexes that could be designated as containing $\mathrm{X}-\mathrm{H} \ldots \pi(\mathrm{X}=\mathrm{C}, \mathrm{N}, \mathrm{O})$ non-covalent bonds, such as benzene...water, benzene...ammonia and benzene...HCN, show significant differences from the conventional hydrogen bonds. Most notably, dispersion contributions are significantly greater than SCF ones. The clear contrast in the origin of stabilisation between the three classes of complex is illustrated in Figure 1.

Figure 1 Illustration of energy partitioning data from Table 1. Vertical dashed lines show separation of classes of complex used in ref. 15. The numbering on the abscissa corresponds to the ordering of the complexes in Table 1. 
Table 2 Partitioned DF-LCCSD interaction energies for selected complexes $\left(\mathrm{kcal} \mathrm{mol}^{-1}\right)$

\begin{tabular}{lccc}
\hline Name & Dispersion & Ionic & $\begin{array}{c}\text { Dispersion- } \\
\text { Exchange }\end{array}$ \\
\hline Ammonia dimer & -1.29 & -0.79 & -0.01 \\
Water dimer & -1.20 & -1.24 & -0.03 \\
Methane dimer & -0.72 & -0.16 & 0.00 \\
Ethene dimer & -1.78 & -0.58 & 0.00 \\
Benzene...methane & -2.03 & -0.82 & +0.01 \\
Ethene...ethyne & -0.95 & -0.54 & 0.00 \\
Benzene....water & -1.90 & -0.99 & -0.01 \\
Benzene....ammonia & -1.96 & -0.85 & -0.01 \\
\hline
\end{tabular}

This analysis was performed at the literature geometries, which were obtained with either MP2 or $\operatorname{CCSD}(\mathrm{T})$ optimisation with triple- or quadruple- $\zeta$ basis sets and including counterpoise correction. 
This can be a laborious process, and might be significantly eased by the advantages of the DFLMP2 method discussed above, particularly the removal of BSSE. Table 3 reports the results of full, unconstrained geometry optimisation of the same S22 complexes using DF-LMP2/aug-ccpVTZ, starting from the literature geometry. Proper application of local correlation methods requires symmetry to be disabled, but the optimisation algorithm retains any elements of symmetry present in the initial geometry.

Optimisation generally proceeded smoothly, in most cases reaching the default convergence criteria in MOLPRO (maximum gradient below $3 \times 10^{-4}$ a.u.) in less than 10 steps. Table 3 demonstrates that the resulting optimised geometries are very similar to their literature equivalents in all but one case, that being the phenol dimer. Performance for hydrogen bonded and dispersion bound complexes is similar, whereas the slightly larger RMS deviation for the mixed complexes is almost entirely due to the phenol dimer (omitting this point gives a mean RMS of $0.018 \AA$ for the remaining six complexes). No significant difference in the quality of agreement is observed between MP2 and $\operatorname{CCSD}(\mathrm{T})$ optimised literature geometries. Figure 2 shows the literature and optimised geometries of the phenol dimer, which by inspection are highly similar. The hydrogen bond O...H distances in the two cases are almost identical (1.937 vs. $1.935 \AA$ ). However, the dihedral angle between the mean planes of each molecule are different, with values of $60.5^{\circ}$ in the literature geometry and $56.3^{\circ}$ in the DF-LMP2 optimised structure. Thus, DF-LMP2 brings the aromatic rings into slightly closer contact (distance between centroids is $5.12 \AA$ vs. $5.39 \AA$ in the literature structure). This subtle change notwithstanding, the overall conclusion is that this efficient and counterpoise-free approach to geometry optimisation satisfactorily and consistently reproduces conventional, corrected geometries.

Table 3 RMS deviations from literature geometry $(\AA)$

\begin{tabular}{lcc}
\hline Name & DF-LMP2 & SCSN \\
\hline Ammonia dimer $^{\mathrm{a}}$ & 0.025 & 0.027 \\
Water dimer $^{\mathrm{a}}$ & 0.030 & 0.037 \\
Formic acid dimer $^{\mathrm{a}}$ & 0.017 & 0.016 \\
Formamide dimer $^{\mathrm{a}}$ & 0.025 & 0.028 \\
Uracil dimer HB & 0.008 & 0.026 \\
2-Pyridoxine...2-aminopyridine & 0.009 & 0.034 \\
Adenine...Thymine HB & 0.011 & 0.028 \\
\hline
\end{tabular}




\begin{tabular}{|c|c|c|}
\hline Methane dimer ${ }^{\mathrm{a}}$ & 0.005 & 0.050 \\
\hline Ethene dimer ${ }^{\mathrm{a}}$ & 0.017 & 0.049 \\
\hline Benzene...methane & 0.005 & 0.039 \\
\hline Benzene dimer stack & 0.029 & 0.068 \\
\hline Pyrazine dimer & 0.021 & 0.048 \\
\hline Uracil dimer stack & 0.023 & 0.053 \\
\hline Indole... benzene stack & 0.017 & 0.039 \\
\hline Adenine...Thymine stack & 0.018 & 0.035 \\
\hline Ethene...ethyne ${ }^{a}$ & 0.014 & 0.017 \\
\hline Benzene...water & 0.026 & 0.012 \\
\hline Benzene....ammonia & 0.019 & 0.021 \\
\hline Benzene...cyanide & 0.019 & 0.016 \\
\hline Benzene dimer $\mathrm{T}$ & 0.015 & 0.027 \\
\hline Indole...benzene $\mathrm{T}$ & 0.012 & 0.023 \\
\hline Phenol dimer & 0.183 & 0.173 \\
\hline Mean & 0.025 & 0.039 \\
\hline Mean H-bond & 0.018 & 0.028 \\
\hline Mean dispersion & 0.017 & 0.048 \\
\hline Mean Mixed & 0.041 & 0.041 \\
\hline Mean $\operatorname{CCSD}(\mathrm{T})^{\mathrm{b}}$ & 0.019 & 0.032 \\
\hline
\end{tabular}

Figure 2 Phenol dimer a) literature geometry; b) DF-LMP2 optmised.

Both conventional MP2 and DF-LMP2 are well known to overestimate the interaction energy of stacked complexes, and might therefore be expected to underestimate intermolecular separation in such complexes. This is apparent in potential energy curves for the stacked benzene dimer, which reach a minimum at vertical separation of $c a .3 .5 \AA$ at $\mathrm{CBS}(\mathrm{T})$ level and $c a .3 .35 \AA$ at $\mathrm{MP} 2{ }^{27}$ Recently, it was demonstrated that a new parameterisation of SCS designed specifically for noncovalent interactions and denoted SCSN, ${ }^{13 \mathrm{a}}$ overcomes this deficiency, resulting in high-accuracy 
interaction energies for all classes of complex with errors of less than $0.5 \mathrm{kcal} \mathrm{mol}^{-1}$. In this approach, correlation energy from same- and opposite-spin electron pairs are weighted by scaling factors in order to correct for systematic errors in standard MP2. The same scaling factors can be applied to calculation of analytic gradients, allowing optimisation to minima on this scaled potential energy surface. Table 3 also reports the RMS deviations from literature geometry using this approach.

In general, SCSN optimised structures are slightly further from literature values than unscaled DFLMP2, both for MP2 and CCSD(T) reference data. Overall performance is similar to unscaled optimisation for hydrogen bonded and mixed complexes, but differences are much larger for dispersion bound complexes. The relatively small RMS deviations reported in Table 3 mask some rather more significant changes; for instance, in the stacked benzene dimer, the separation of mean planes is $3.361 \AA$ in the literature (MP2/TZ-CP optimised) geometry, whereas our SCSN optimisation results in a separation of $3.487 \AA$, much closer to the minimum of the CBS(T) curve. ${ }^{27}$ A similar trend is apparent in other stacked complexes, albeit to a lesser extent: in the pyrazine dimer, the molecular planes are separated by $3.261 \AA$ in literature geometry and $3.270 \AA$ in the SCSN geometry. In stacked adenine-thymine, molecular planes are not quite parallel, so for the current investigation the perpendicular distance is defined as that from the mean plane of adenine to the centroid of the 6-membered ring of thymine, which is $3.174 \AA$ in literature and $3.205 \AA$ in SCSN optimised geometry. Thus, it is evident that the excess stabilisation of stacked complexes at MP2 level carries through into potential energy surfaces, and can be simply and efficiently corrected by the empirical SCSN scheme.

A second SCSN geometry optimisation was performed for the T-shaped benzene dimer, following the recent study by Wang et al. that showed the lowest energy geometry of this complex to deviate from the ideal $C_{2 \mathrm{v}}$ geometry. ${ }^{28}$ Starting from a slightly offset geometry, optimisation proceeded smoothly to a point just $0.06 \mathrm{kcal} \mathrm{mol}^{-1}$ lower in energy than that reported in Table 3 . Shown in Figure 3, the SCSN geometry is similar to that reported in ref.28, with the donor $\mathrm{C}-\mathrm{H}$ directed towards a $\mathrm{C}-\mathrm{C}$ bond rather than the centre of the acceptor ring.

Figure 3 SCSN optimised geometry of T-shaped benzene dimer 
A similar procedure was followed for the SCSN-optimised geometries. In this case, both the $\mathrm{C}_{2 \mathrm{v}}$ and $\mathrm{C}_{\mathrm{s}}$ optimised forms of the $\mathrm{T}$-shaped benzene dimer are found to be true minima, echoing the findings of Wang et $a l,{ }^{28}$ who used dispersion-corrected DFT to show that both forms are stable and are connected by a transition state with a barrier of just $0.01 \mathrm{kcal} \mathrm{mol}^{-1}$. In this regard, SCSN again seems to be slightly more accurate than unscaled DF-LMP2 in finding both forms to be stable, but the inherent errors associated with this approach are much larger than these energy differences, such that any conclusion based on this data must be tentative at best. All remaining structures are found to be minima using this approach. Two geometries of the stacked (paralleldisplaced) benzene dimer were tested, one with a $\mathrm{C}-\mathrm{H}$ bond situated over the partner benzene and a second with a $\mathrm{C}-\mathrm{C}$ bond over the other benzene. Both are found to be minima using SCSN, with effectively identical energies within the expected accuracy of the method (less than $0.1 \mathrm{kcal}$ $\mathrm{mol}^{-1}$ difference).

As well as confirming structures as minima, harmonic frequency calculations can be used to estimate the change in zero-point vibrational energy (ZPVE) that accompanies formation of a complex from constituent monomers. Such changes stem largely from conversion of translational and rotational degrees of freedom of individual molecules into intermolecular vibrations. Such vibrations are likely to be strongly anharmonic in nature, such that harmonic data can only be a first-order approximation to the true ZPVE correction. Nonetheless, obtaining even harmonic vibrational data for the larger complexes considered here is a considerable challenge, and the data presented in Table 4 is a step towards establishing accurate ZPVE corrections for systems of this size. 
Table 4 Harmonic zero point energy corrections for binding $\left(\mathrm{kcal} \mathrm{mol}^{-1}\right)$

\begin{tabular}{|c|c|c|c|}
\hline Name & DF-LMP2 & Scaled $^{\mathrm{a}}$ & SCSN \\
\hline Ammonia dimer & 1.70 & 1.62 & 1.66 \\
\hline Water dimer & 2.37 & 2.27 & 2.33 \\
\hline Formic acid dimer & 2.08 & 1.99 & 2.06 \\
\hline Formamide dimer & 2.64 & 2.53 & 2.52 \\
\hline Uracil dimer HB & 1.21 & 1.16 & 1.15 \\
\hline 2-Pyridoxine...2-aminopyridine & 1.01 & 0.97 & 0.98 \\
\hline Adenine...thymine HB & 0.95 & 0.90 & 0.89 \\
\hline Methane dimer & 1.16 & 1.11 & 1.14 \\
\hline Ethene dimer & 0.83 & 0.80 & 0.82 \\
\hline Benzene...methane & 0.64 & 0.62 & 0.70 \\
\hline Benzene dimer stack & 0.53 & 0.51 & 0.56 \\
\hline Pyrazine dimer & 0.61 & 0.58 & 0.51 \\
\hline Uracil dimer stack & 0.58 & 0.56 & 0.56 \\
\hline Indole...benzene stack & 0.25 & 0.24 & 0.43 \\
\hline Adenine...thymine stack & 0.72 & 0.68 & 0.61 \\
\hline Ethene...ethyne & 0.71 & 0.68 & 0.73 \\
\hline Benzene...water & 1.06 & 1.01 & 1.04 \\
\hline Benzene...ammonia & 0.70 & 0.67 & 0.79 \\
\hline Benzene...cyanide & 0.60 & 0.57 & 0.71 \\
\hline Benzene dimer $\mathrm{T}$ & 0.52 & 0.49 & 0.67 \\
\hline Indole...benzene $\mathrm{T}$ & 0.62 & 0.60 & 0.59 \\
\hline Phenol dimer & 0.95 & 0.91 & 0.99 \\
\hline
\end{tabular}

${ }^{\mathrm{a}}$ Scaled from raw DF-LMP2 data using the recommended scaling factor of 0.956

$\triangle$ ZPVE values on formation of complexes from monomers are largest for the hydrogen bonded complexes, and are in general rather smaller for dispersion bound and mixed complexes. The single largest change is found for the formamide dimer, closely followed by water and formic acid dimers. The value for the water dimer is similar to previous estimates using MP2 methods with the harmonic approximation $\left(2.1-2.3 \mathrm{kcal} \mathrm{mol}^{-1}\right)$, and as expected rather larger than values that take anharmonicity into account $\left(1.8-2.1 \mathrm{kcal} \mathrm{mol}^{-1}\right){ }^{30}$ The larger aromatic systems containing two 


\section{Conclusions}

The utility of local correlation methods, when combined with density fitting, has been demonstrated in the study of non-covalent interactions. By construction, these methods allow for decomposition of correlation energy into contributions from dispersion and ionic excitations, thus giving insight into the origin of stabilisation at no additional computational cost to the correlation calculation. This analysis, at both DF-LMP2 and DF-LCCSD levels, clearly distinguishes complexes bound by hydrogen bonding, dispersion, or a mixture of these effects. The lack of basis set superposition error in local methods, coupled with their computational efficiency, makes them ideally suited for simple geometry optimisation and harmonic frequency calculation. Geometry optimisation leads to structures in close agreement with published, counterpoise-corrected geometries, demonstrating the utility of this approach. Harmonic frequency analysis, using both unscaled and SCSN-scaled MP2 data, indicates that most complexes within the S22 set are at least local minima on their respective potential energy surfaces. The only exception to this conclusion is the $\mathrm{C}_{2 \mathrm{v}}$ T-shaped benzene dimer, which is a minimum with SCSN scaling but a saddle point with unscaled MP2. Zero-point vibrational energy corrections to binding energies have also been 
estimated, using raw data and two distinct scaling methods. In general, all three methods are in reasonable agreement, with corrections of between $20 \%$ and $50 \%$ of uncorrected binding energies.

\section{Acknowledgements}

This work was funded by the UK EPSRC, grant ref. EP/C013328/1.

\section{References}

${ }^{1}$ A. Pavlov, P.M. Mitrasinovic, Curr. Org. Chem., 2010, 14, 129.

${ }^{2}$ P. Hobza, H.L. Selzle, E.W. Schlag, J. Phys. Chem., 1996, 100, 18790.

${ }^{3}$ M. Schütz, G. Rauhut, H.-J. Werner, J. Phys. Chem. A, 1998, 102, 5997.

${ }^{4}$ H.-J. Werner, F. R. Manby, P. J. Knowles, J. Chem. Phys., 2003, 118, 8149.

${ }^{5}$ C. Hampel, H.-J. Werner, J. Chem. Phys., 1996, 104, 6286.

${ }^{6}$ M. Schütz, H.-J. Werner, J. Chem. Phys., 2001, 114, 661.

${ }^{7}$ M. Schütz, Phys. Chem. Chem. Phys., 2002, 4, 3941.

${ }^{8}$ M. Schütz, F. R. Manby, Phys. Chem. Chem. Phys., 2003, 5, 3349.

${ }^{9}$ S. F. Boys, F. Bernardi, Mol. Phys., 1970, 19, 553.

${ }^{10}$ B. Jeziorski, R. Moszynski, K. Szalewicz, Chem. Rev., 1994, 94, 1887.

${ }^{11}$ T. Hrenar, G. Rauhut, H.-J. Werner, J. Phys. Chem. A, 2006, 110, 2060.

${ }^{12}$ S. Grimme, J. Chem. Phys., 2003, 118, 9095.

13 a) J.G. Hill, J.A. Platts, J. Chem. Theor. Comput., 2007, 3, 80. b) R.A. Bachorz, F. A. Bischoff, S. Hofener, W. Klopper, P. Ottiger, R. Leist, J. A. Frey, S. Leutwyler, Phys. Chem. Chem. Phys., 2008, 10, 2758.

${ }^{14}$ O. Marchetti and H.-J. Werner, J. Phys. Chem. A, 2009, 113, 11580.

${ }^{15}$ P. Jurečka, J. Šponer, J. Černý, P. Hobza, Phys. Chem. Chem. Phys., 2006, 8, 1985.

${ }^{16}$ MOLPRO, version 2008.1, a package of ab initio programs, H.-J. Werner, P. J. Knowles, R. Lindh, F. R. Manby, M. Schütz and others, see http://www.molpro.net.

${ }^{17}$ T. Dunning Jr., J. Chem. Phys., 1989, 90, 1007.

${ }^{18}$ R. A. Kendall, T. Dunning Jr., R. J. Harrison, J. Chem. Phys., 1991, 96, 6797.

${ }^{19}$ F. Weigend, A. Köhn, C. Hättig, J. Chem. Phys., 2002, 116, 3175.

${ }^{20}$ R. Polly, H.-J. Werner, F. R. Manby, P. J. Knowles, Mol. Phys., 2004, 102, 2311.

${ }^{21}$ F. Weigend, Phys. Chem. Chem. Phys., 2002, 4, 4285.

${ }^{22}$ J. Pipek, P. G. Mezey, J. Chem. Phys., 1989, 90, 4916.

${ }^{23}$ J. W. Boughton, P. Pulay, J. Comput. Chem., 1993, 14, 736. 
${ }^{24}$ M. Schütz, H.-J. Werner, R. Lindh, F. R. Manby, J. Chem. Phys., 2004, 121, 737.

${ }^{25}$ Chemcraft v1.6: www.chemcraftprog.com

${ }^{26}$ J. Neugebauer, M. Reiher, C. Kind, B.A. Hess, J. Comput. Chem., 2002, 23, 895.

27 a) S. Tsuzuki, K. Honda, T. Uchimaru, M. Mikami, K. Tanabe, J. Am. Chem. Soc., 2000, 122, 3746. b) M. O. Sinnokrot, C. D. Sherrill, J. Am. Chem. Soc., 2004, 126, 7690. c) J.G. Hill, J.A. Platts, H.-J. Werner, Phys. Chem. Chem. Phys., 2006, 8, 4072.

${ }^{28}$ W. Wang, M. Pitoňák, P. Hobza, ChemPhysChem, 2007, 8, 2107.

${ }^{29}$ K. Gkionis, J.G. Hill, S.P. Oldfield, J.A. Platts, J. Mol. Model., 2009, 15, 1051.

${ }^{30}$ M. W. Feyereisen, D. Feller, D.A. Dixon, J. Phys. Chem., 1996, 100, 2993. 